\title{
SOME NEW OBSERVATION ON INVARIANT THEORY OF PLANE QUARTICS*
}

\author{
TETSUJI SHIODA ${ }^{\dagger}$
}

1. Introduction. Let $S(n, m)$ denote the graded ring of projective invariants of an $n$-ary form (a homogeneous polynomial in $n$ variables) of degree $m$. We are interested in the case $n=3$ and $m=4$. A ternary quartic form $F\left(x_{0}, x_{1}, x_{2}\right)$ defines a plane curve of genus 3 if it is nonsingular, and conversely any non-hyperelliptic curve of genus 3 can be realized as such a plane quartic via the canonical embedding, which is unique up to projective transformations. Thus the structure of the ring $S(3,4)$ is closely related to the moduli of genus 3 curves. (For general background of Invariant Theory, see e.g. [4], [13].)

More than thirty years ago ([5, Appendix $]$ ), we calculated the generating function (Poincaré series) of $S(3,4)$ and made a few guess (or conjecture?) about the structure of the graded ring $S(3,4)$. More recently, Dixmier [2] has proved the existence of a system of parameters for this ring (suggested in [5]) by exhibiting a system of seven explicit projective invariants.

In this paper, we study some close relationship of the ring $S(3,4)$ of projective invariants to another invariant theory, i.e. to the invariant theory for the Weyl groups $W\left(E_{7}\right)$ and $W\left(E_{6}\right)$ (cf. [1]). We are led to such a connection from the viewpoint of Mordell-Weil lattices ([8], [9]).

2. Formulation of main results. We consider the case of characterisitic zero. Taking

$$
F\left(x_{0}, x_{1}, x_{2}\right)=\sum_{i_{0}+i_{1}+i_{2}=4} a_{i_{0}, i_{1}, i_{2}} x_{0}^{i_{0}} x_{1}^{i_{1}} x_{2}^{i_{2}}
$$

with variable coefficients $\left\{a_{i_{0}, i_{1}, i_{2}}\right\}$, we may regard $S(3,4)$ as a graded subring of the polynomial ring $\mathbf{C}\left[a_{i_{0}, i_{1}, i_{2}}\right]$ graded by the total degree, consisting of those $I=$ $I(F) \in \mathbf{C}\left[a_{i_{0}, i_{1}, i_{2}}\right]$ which are invariant under $S L(3)$. Namely, for any $g \in S L(3)$, let $\left(x_{0}^{\prime}, x_{1}^{\prime}, x_{2}^{\prime}\right)=\left(x_{0}, x_{1}, x_{2}\right) g$ and rewrite $F\left(x_{0}^{\prime}, x_{1}^{\prime}, x_{2}^{\prime}\right)$ as a polynomial $F^{\prime}\left(x_{0}, x_{1}, x_{2}\right)$ in $x_{0}, x_{1}, x_{2}$ :

$$
F^{\prime}\left(x_{0}, x_{1}, x_{2}\right)=\sum_{i_{0}+i_{1}+i_{2}=4} a_{i_{0}, i_{1}, i_{2}}^{\prime} x_{0}^{i_{0}} x_{1}^{i_{1}} x_{2}^{i_{2}}
$$

We set $F^{g}=F^{\prime}$. Then, by definition, we have

$$
I \in S(3,4) \Longleftrightarrow I\left(F^{g}\right)=I(F)(\forall g \in S L(3)) .
$$

For any ternary quartic form $F_{0}$, we call the map $I \rightarrow I\left(F_{0}\right)$ the evaluation map of $S(3,4)$ at $F_{0}$.

*Received December 9, 1999; accepted for publication February 9, 2000.

tDepartment of Mathematics, Rikkyo University, Nishi-Ikebukuro, Toshima-ku, Tokyo 171, Japan (shioda@rkmath.rikkyo.ac.jp). 
Actually the C-algebra $S(3,4)$ is obtained from the Q-algebra $S(3,4) \cap \mathbf{Q}\left[a_{i_{0}, i_{1}, i_{2}}\right]$ by the scalar extension of $\mathbf{Q}$ to $\mathbf{C}$. So, in the following, we change the notation so that $S(3,4)$ will denote this $\mathbf{Q}$-subalgebra of $\mathbf{Q}\left[a_{i_{0}, i_{1}, i_{2}}\right]$

Now we recall the following fact on the normal form of a plane quartic with a given flex (cf. $[8, \S 1])$. Take the inhomogeneous coordinates $x, t$ such that $\left(x_{0}: x_{1}\right.$ : $\left.x_{2}\right)=(1: x: t)$. The normal form of type $E_{7}$ is

$$
f_{\lambda}=x^{3}+x\left(p_{0}+p_{1} t+t^{3}\right)+q_{0}+q_{1} t+q_{2} t^{2}+q_{3} t^{3}+q_{4} t^{4}
$$

with $\lambda=\left(p_{0}, p_{1}, q_{0}, \ldots, q_{4}\right) \in \mathbf{A}^{7}$, and the normal form of type $E_{6}$ is

$$
f_{\lambda}=x^{3}+x\left(p_{0}+p_{1} t+p_{2} t^{2}\right)+q_{0}+q_{1} t+q_{2} t^{2}+t^{4}
$$

with $\lambda=\left(p_{0}, p_{1}, p_{2}, q_{0}, q_{1}, q_{2}\right) \in \mathbf{A}^{6}$. In either case, let $\Gamma_{\lambda}$ be the plane quartic defined by $f_{\lambda}=0$; the flex is given by the point $\left(x_{0}: x_{1}: x_{2}\right)=(0: 1: 0)$. The fact is that every plane quartic with a given flex is isomorphic to $\Gamma_{\lambda}$ for some $\lambda \in \mathbf{A}^{7}$ or $\mathbf{A}^{6}$; the distinction depends on whether the given flex is ordinary or special ${ }^{1}$ (i.e. whether the tangent line to the curve at the flex intersects the curve with multiplicity 3 or 4 ).

It is obvious that the evaluation map $I \rightarrow I\left(f_{\lambda}\right)$ gives a ring homomorphism

$$
\phi: S(3,4) \rightarrow \mathrm{Q}[\lambda]=\mathrm{Q}\left[p_{i}, q_{j}\right]
$$

for either type of $f_{\lambda}$. Let us call it the evaluation map of type $E_{7}$ or $E_{6}$, and denote it by $\phi_{7}$ or $\phi_{6}$ when we need to specify the cases.

The main purpose of this paper is to establish less obvious relationship between the invariant theory of a plane quartic and the invariant theory of the Weyl group $W\left(E_{r}\right)(r=6,7)$. To formulate the results, note first that the ring of invariants of $W\left(E_{r}\right)$, say $R\left(E_{r}\right)$, can be naturally identified with $\mathbf{Q}[\lambda]$ given above (see [1], [6], [7]), which is a graded polynomial ring with the weights of $p_{i}$ or $q_{j}$ assigned as follows:

for $E_{7}$ case: $w t\left(p_{i}\right)=12-4 i, w t\left(q_{j}\right)=18-4 j$.

for $E_{6}$ case: $w t\left(p_{i}\right)=8-3 i, w t\left(q_{j}\right)=12-3 j$.

On the other hand, let

$$
S=S(3,4)=\oplus_{m} S_{m}
$$

where $S_{m}$ is the homogeneous part of degree $m$ of $S$. It is known that $S_{m} \neq 0$ only if $m$ is a multiple of 3 (cf. §3).

THEOREM 1. (i) The evaluation map of type $E_{7}$

$$
\phi_{7}: S(3,4) \longrightarrow R\left(E_{7}\right)=\mathbf{Q}\left[p_{0}, p_{1}, q_{0}, q_{1}, q_{2}, q_{3}, q_{4}\right]
$$

is a graded homomorphism from $S(3,4)$ to $R\left(E_{7}\right)$ with weight ratio $3: 14$ in the sense that $\phi$ sends $S_{3 d}$ to $R\left(E_{7}\right)_{14 d}$ for all $d$.

(ii) The evaluation map of type $E_{6}$

$$
\phi_{6}: S(3,4) \longrightarrow R\left(E_{6}\right)=\mathrm{Q}\left[p_{0}, p_{1}, p_{2}, q_{0}, q_{1}, q_{2}\right]
$$

\footnotetext{
${ }^{1}$ see the comments at the end of the paper.
} 
is a graded homomorphism from $S(3,4)$ to $R\left(E_{6}\right)$ with weight ratio $3: 8$ in a similar sense.

THEOREM 2. Let $D \in S(3,4)$ denote the discriminant of a plane quartic: its characteristic property is that $D \in S_{27}$ and $D(f) \neq 0$ if and only if $f=0$ is smooth. Then the image $\phi(D)$ under the evaluation map $\phi$ of type $E_{r}(r=7,6)$ is equal, up to a constant, to the "discriminant" $\delta$ of $R\left(E_{r}\right)$ which is defined as the square of the basic anti-invariant of $W\left(E_{r}\right)$; the weight of $\delta$ is 126 or 72 for $r=7$ or 6 .

THEOREM 3. (i) For $r=7$, the evaluation map $\phi_{7}$ is injective.

(ii)For $r=6$, the evaluation map $\phi_{6}$ has a nontrivial kernel which contains a projective invariant $J$ of degree $60 .^{2}$

For a graded integral domain $R, F(R)$ will denote the field of fractions of $R$, and $F(R)_{(0)}$ will denote the subfield of homogeneous fractions (i.e. the fractions $a / b$ with $a, b \in R$ of the same weight).

For $S=S(3,4), F(S)_{(0)}$ can be considered as the function field of the moduli space $\mathcal{M}_{3}$ of curves of genus 3 .

TheOREM 4. Let $P=\mathrm{Q}\left[I_{1}, \ldots, I_{6}, I_{9}\right]$ be the polynomial subring of $S=S(3,4)$ generated by the Dixmier's system $\left\{I_{d}(d=1, \ldots, 6,9)\right\}, I_{d}$ being a suitable projective invariant of degree $3 d$. Then we have the algebraic extensions

$$
F(P)_{(0)} \subset F(S)_{(0)} \subset F(\mathbf{Q}[\lambda])_{(0)}
$$

with the extension degree

$$
\left[F(S)_{(0)}: F(P)_{(0)}\right]=50, \quad\left[F(\mathbf{Q}[\lambda])_{(0)}: F(S)_{(0)}\right]=24 .
$$

REMARK. (1) Note that both

$$
F(P)_{(0)}=\mathrm{Q}\left(I_{d} / I_{1}^{d}(d=1, \ldots, 6,9)\right)
$$

and

$$
F(\mathbf{Q}[\lambda])_{(0)}=\mathbf{Q}\left(p_{0} / q_{4}^{6}, p_{1} / q_{4}^{4}, q_{0} / q_{4}^{9}, q_{1} / q_{4}^{7}, q_{2} / q_{4}^{5}, q_{3} / q_{4}^{3}\right)
$$

are rational fields (i.e. purely transcendental extensions) over $\mathbf{Q}$. The famous rationality question of the moduli space $\mathcal{M}_{3}$ of curves of genus 3 is equivalent to asking whether $F(S)_{(0)}$ is a rational field or not. This was answered by Katsylo [3] by a representation-theoretic method. Our approach might be of some use to this question, from a more geometric point of view.

(2) The explicit form of the invariants $I_{d}$ in the Dixmier's system is not necessary to prove Theorem 4, but we shall give it in [11] for a possible use in future.

3. Proof of Theorems. We keep the notation introduced in the above.

First recall that, for any homogeneous invariant $I \in S=S(3,4)$ of degree $m$ $\left(I \in S_{m}\right)$, we have

$$
I\left(F^{g}\right)=\operatorname{det}(g)^{w} I(F)(\forall g \in G L(3))
$$

\footnotetext{
${ }^{2}$ see the comments at the end of the paper
} 
for some integer $w$, which is determined by $4 m=3 w$ (by comparing the degree in generic coefficients of $g$ ). Thus, if $I \neq 0, m=3 d$ and $w=4 d$ for some integer $d$.

Proof of Theorem 1. The key point is the weighted homogeneity of $f_{\lambda}$. For the normal form of type $E_{7}, f_{\lambda}$ is a weighted homogeneous polynomial of total weight 18, if we fix $w t(x)=6$ and $w t(t)=4$. Namely we have

$$
f_{\lambda^{\prime}}\left(u^{6} x, u^{4} t\right)=u^{18} f_{\lambda}(x, t) \quad\left(\forall u \in \mathbf{G}_{m}\right)
$$

with $\lambda^{\prime}=\left(u^{12} p_{0}, u^{8} p_{1}, \ldots, u^{6} q_{3}, u^{2} q_{4}\right)$.

Let $g$ be the diagonal matrix $g=\left[1, u^{6}, u^{4}\right] \in G L(3)$; note $\operatorname{det}(g)=u^{10}$. Then we have from the above

$$
\left(f_{\lambda^{\prime}}\right)^{g}(x, t)=u^{18} f_{\lambda}(x, t)
$$

For any $I \in S_{3 d}$, we have then

$$
\left(u^{10}\right)^{4 d} I\left(f_{\lambda^{\prime}}\right)=\left(u^{18}\right)^{3 d} I\left(f_{\lambda}\right)
$$

which implies

$$
I\left(f_{\lambda^{\prime}}\right)=u^{14 d} I\left(f_{\lambda}\right) \quad\left(\forall u \in \mathbf{G}_{m}\right)
$$

This proves that $\phi_{7}(I)=I\left(f_{\lambda}\right)$ has weight $14 d$ for any $I \in S_{3 d}$. Thus part (i) of Theorem 1 is shown.

For the normal form of type $E_{6}, f_{\lambda}$ is a weighted homogeneous polynomial of total weight 12 by taking $w t(x)=4$ and $w t(t)=3$. The same argument as above shows part (ii) of Theorem 1.

Proof of Theorem 2. Since the discriminant $D$ of a plane quartic has degree 27 $\left(D \in S_{27}\right), \phi_{7}(D)$ has weight $9 \cdot 14=126$ and $\phi_{6}(D)$ has weight $9 \cdot 8=72$ by Theorem 1. Hence $\phi(D) \in \mathbf{Q}[\lambda]$ has the same weight as the discriminant $\delta$ of $R\left(E_{r}\right)$ (= the number of the roots in $E_{r}$ ) for $r=7,6$.

To prove $\phi(D)=\delta$ (up to a constant), the simplest would be to assume the knowledge of singularity theory. From this standpoint, note first that the plane quartic $\Gamma_{\lambda}$ is smooth at the points at infinity (i.e. on $x_{0}=0$ ). Thus it will be smooth if and only if the affine curve $f_{\lambda}=0$ is smooth. By Jacobian criterion, the latter condition is equivalent to the smoothness of the affine surface $S_{\lambda}^{\prime}: y^{2}=f_{\lambda}$ (since char $\neq 2$ ).

Now the singularity theory tells us that the family $y^{2}=f_{\lambda}$ parametrized by $\lambda \epsilon$ $\mathrm{A}^{7}$ is a so-called semi-universal deformation of the $E_{r}$-singularity $y^{2}=x^{3}+x t^{3}(r=7)$ or $y^{2}=x^{3}+t^{4}(r=6)$ and that $S_{\lambda}^{\prime}$ is smooth if and only if $\delta(\lambda) \neq 0$.

Therefore we have $\phi(D) \neq 0 \Leftrightarrow \delta(\lambda) \neq 0$, proving the assertion.

We give here an alternative proof based on the theory of Mordell-Weil lattices (MWL) (cf. [6], [7], esp. [8, Th.5]). We consider the elliptic curve

$$
E=E_{\lambda}: y^{2}=f_{\lambda}=x^{3}+\cdots
$$

defined over $K=k(t), k$ being the algebraic closure of $\mathbf{Q}\left(p_{i}, q_{j}\right)$. To fix the idea, suppose $f_{\lambda}$ is of type $E_{7}$ and $\lambda$ is generic over $\mathbf{Q}$ (i.e. $p_{i}, q_{j}$ are algebraically independent over $\mathbf{Q})$. Then the structure of the Mordell-Weil lattice $E(K)$ is isomorphic to $E_{7}^{*}$, 
the dual lattice of the root lattice $E_{7}$, with the narrow Mordell-Weil lattice $E(K)^{0}$ being isomorphic to $E_{7}$. Corresponding to the 56 minimal vectors of norm $3 / 2$ in $E_{7}^{*}$, there are $56 k(t)$-rational points $P=(x, y)$ of the form:

$$
x=a t+b, \quad y=c t^{2}+d t+e
$$

([6], Lemma 9.1). A nice fact is that the map $P \mapsto c$ extends to a group homomorphism $s p: E(K) \rightarrow k$ (the specialization map at $t=\infty$, up to a constant), which is injective for $\lambda$ generic.

We can choose $\left\{P_{1}, \ldots, P_{7}\right\} \subset E(K)$ such that $\left\langle P_{i}, P_{j}\right\rangle=\delta_{i j}+1 / 2$ (see [8], [10]); they generate a subgroup of index 3 in $E(K)$. Then $c_{i}=s p\left(P_{i}\right) \in k(i=$ $1, \ldots, 7)$ are algebraically independent over $\mathbf{Q}$, and the Weyl group $W\left(E_{7}\right)$ acts on the polynomial ring $\mathbf{Q}\left[c_{1}, \ldots, c_{7}\right]$ in such a way that the ring of invariants is equal to $\mathbf{Q}\left[p_{0}, p_{1}, q_{0}, \ldots, q_{4}\right]$. Moreover the coefficients $a, b, \ldots, e$ defining $P=(x, y)$ belong to $\mathbf{Q}\left[c_{1}, \ldots, c_{7}\right]$ for all $P$.

The basic anti-invariant in $\mathbf{Q}\left[c_{i}\right]$ is the product of 63 linear forms:

$$
c_{i}-c_{j}(i<j), c_{i}-v, v-c_{i}-c_{j}-c_{k}(i<j<k)
$$

where $v=\left(\sum_{i} c_{i}\right) / 3$, which are the image of half of the 126 roots in $E(K)^{0} \simeq E_{7}$. The discriminant $\delta(\lambda)$ is the square of this anti-invariant up to a constant.

Now we consider specializing the generic parameter $\lambda$ to any $\lambda^{\prime} \in \mathbf{A}^{7}$. If the MWL does not degenerate under this specialization, we have the 126 roots in $E_{\lambda^{\prime}}(K)^{0} \simeq E_{7}$. Recall that a root in $E_{7}$ corresponds to a rational point $Q=(x, y)$ of the form

$$
x=t^{2} / u^{2}+a t+b, \quad y=t^{3} / u^{3}+c t^{2}+d t+e
$$

with $u=s p(Q) \neq 0$. Therefore none of the 63 linear forms above corresponding to the roots vanish under the specialization, and we have $\delta\left(\lambda^{\prime}\right) \neq 0$. In other words, $\delta\left(\lambda^{\prime}\right)=0$ implies the degeneration of MWL (this is the MWL-analogue of "vanishing cycles" in the singularity theory).

Further note that the degeneration of MWL occurs if and only if the affine surface $S_{\lambda^{\prime}}$ acquires singularities, since both conditions are equivalent to the existence of a reducible fibre in the associated elliptic fibration at $t \neq \infty$.

Thus we have the implication $\delta\left(\lambda^{\prime}\right)=0 \Rightarrow D\left(\lambda^{\prime}\right)=0$. Comparing the degree, we conclude that $\delta=\phi(D)$ up to a constant.

The case of $E_{6}$ can be treated in a similar way:

REMARK. It is also possible to directly verify $\dot{\phi}(D)=\delta$ (up to a constant) by means of computer algebra (cf. [11]).

Proof of Theorem 3. The injectivity of the homomorphism $\phi_{7}$ is clear, because a generic plane quartic can be put in the normal form $\Gamma_{\lambda}: f_{\lambda}=0$ (over a field of rationality of the curve and a flex $)([8, \S 1])$.

To prove the second part, we use the notation in the above proof of Theorem 2. For each of the $56 k(t)$-rational points $P=(x, y) \in E_{\lambda}$, we have the identity in $t$ :

$$
\left(c t^{2}+d t+e\right)^{2}=f_{\lambda}(a t+b, t) .
$$


This means that the line $L: x=a t+b$ in $\mathbf{P}^{2}$ is a bitangent to the plane quartic $\Gamma_{\lambda}: f_{\lambda}=0$, i.e. we have $L \cdot \Gamma_{\lambda}=2 A+2 B$ for the two points $A, B \in \Gamma_{\lambda}$, which are determined by the equation $c t^{2}+d t+e=0$. In this way, we get all the 28 bitangents to $\Gamma_{\lambda}$, since $\pm P=(x, \pm y)$ give the same bitangent.

Consider the product

$$
J=\prod_{\nu=1}^{28}\left(d_{\nu}^{2}-4 c_{\nu} e_{\nu}\right)
$$

which is an element of $\mathbf{Q}\left[c_{1}, \ldots, c_{7}\right]$ of weight $28 \cdot 10=280$. Since the Weyl group $W\left(E_{7}\right)$ acts (transitively) on the 56 minimal vectors, $J$ is an invariant. Hence

$$
J \in \mathbf{Q}\left[c_{1}, \ldots, c_{7}\right]^{W\left(E_{7}\right)}=\mathbf{Q}\left[p_{0}, p_{1}, q_{0}, \ldots, q_{4}\right] .
$$

LEMMA 5. For the normal form of type $E_{7}$, the vanishing of the invariant $J$ is equivalent to the existence of a special flex.

Proof Assume $J\left(\lambda^{\prime}\right)=0$ for $\lambda^{\prime} \in \mathbf{A}^{7}$. Then some factor in the product must be 0 , so the two points of contact of a bitangent coincide. In other words, we have

$$
L \cdot \Gamma_{\lambda^{\prime}}=4 A
$$

for this bitangent $L$. Then this point $A$ is a special flex of $\Gamma_{\lambda^{\prime}}$ with flex tangent $L$. The converse is clear.

By the lemma, the vanishing of $J$ has an invariant meaning in the sense of projective geometry. Hence $J$ is a projective invariant, or more precisely, we have $J=\phi_{7}(I)$ for a unique projective invariant $I \in S(3,4)$. In view of Theorem $1, I$ has degree 60 . Finally $I$ belongs to the kernel of the map $\phi_{6}$, since the normal form of type $E_{6}$ has a special flex by definition.

This complete the proof of Theorem 3.

Presumably the invariant $I$ constructed above should be the generator of $\operatorname{Ker}\left(\phi_{6}\right)$, but it is not yet proven.

Proof of Theorem 4. By [2], $P$ is a polynomial subring of $S$ such that $S$ is integral over $P$. Then by [5, Lemma 1], $F(S)$ is an algebraic extension of $F(P)$ of degree $N(1)$ if $N(T)$ denotes the numerator of the generating function of $S$. In our case ([5, Appendix $])$, the generating function is equal to

$$
\frac{N(T)}{\prod_{d=1}^{6}\left(1-T^{d}\right) \cdot\left(1-T^{9}\right)} \quad\left(T=t^{3}\right)
$$

with

$N(T)=1+T^{3}+T^{4}+T^{5}+2 T^{6}+3 T^{7}+2 T^{8}+3 T^{9}+4 T^{10}+3 T^{11}+4 T^{12}+4 T^{13}+$ $3 T^{14}+4 T^{15}+3 T^{16}+2 T^{17}+3 T^{18}+2 T^{19}+T^{20}+T^{21}+T^{22}+T^{25}$.

Hence we have $N(1)=50$, which shows $[F(S): F(P)]=50$. It follows easily that we have $\left[F(S)_{(0)}: F(P)_{(0)}\right]=50$.

On the other hand, we view $F(S)$ as a subfield of $\mathbf{Q}\left(p_{0}, p_{1}, q_{0}, \ldots, q_{4}\right)$ via the injective map $\phi_{7}$ (Theorem 3). Suppose $\Gamma$ is a generic plane quartic. Then it has 
24 flexes, say $\xi_{\nu}$, which are all ordinary flexes. For each choice of the flex $\xi_{\nu}, \Gamma$ is isomorphic to $\Gamma_{\lambda}$ for some $\lambda=\left(p_{i}, q_{j}\right) \in \mathbf{A}^{7}$, with $\xi_{\nu}$ mapped to $(0,1,0) \in \Gamma_{\lambda}$; moreover $\lambda=\lambda^{(\nu)}$ is uniquely determined by the condition $q_{4}=1$ for the given pair $\left(\Gamma, \xi_{\nu}\right)$ (see $\left.[8, \S 1]\right)$. Thus the 24 values of $\lambda^{(\nu)}$ corresponding to the 24 flexes are mutually conjugate over $F(S)_{(0)}$. It follows that $\left[F(\mathbf{Q}[\lambda])_{(0)}, F(S)_{0}\right]=24$.

AcKnowledgement. The author owes the following valuable remarks to the referee:

(1) about the terminology. A point of undulation is a more standard word for a special flex used in this paper and [8]. See Salmon's book [12], no. 50, p. 37 and no. 247 , p. 218.

(2) about a characterization of undulation. Theorem 3 (ii) is classically known, and is a special case of the following fact. Salmon describes a projective invariant of degree $6(m-3)(3 m-2)$ for a plane curve of degree $m$ whose vanishing expresses the condition that the curve has a point of undulation ([12], no. 400, p. 362).

\section{REFERENCES}

[1] N. Bourbaki, Groupes et Algèbres de Lie, Chap. 4,5 et 6, Hermann, Paris, 1968; Masson, 1981.

[2] J. DiXmIER, On the projective invariants of quartic plane curves, Adv. in Math., 64 (1987), pp. 279-304.

[3] P. Katsylo, Rationality of the moduli variety of curves of genus 3, Commemt. Math. Helv., 71 (1996), pp. 507-524.

[4] D. Mumford, Geometric Invariant Theory, Springer-Verlag, 1965; 3rd ed. (with J. Fogarty, F. Kirwan), 1994.

[5] T. SHIODA, On the graded ring of invariants of binary octavics, Am. J. Math., 89 (1967), pp. 1022-1046.

[6] - Construction of elliptic curves with high rank via the invariants of the Weyl groups, J. Math. Soc. Japan, 43 (1991), pp. 673-719.

[7] - Theory of Mordell-Weil lattices, in Proc. ICM Kyoto-1990, vol. I, 1991, pp. 473-489.

[8] - Plane quartics and Mordell-Weil lattices of type $E_{7}$, Comment. Math. Univ. St. Pauli, 42 (1993) , pp. 61-79.

[9] - Weierstrass transformations and cubic surfaces, Comment. Math. Univ. St. Pauli, 44 (1995), pp. 109-128.

$[10]-, A$ uniform construction of the root lattices $E_{6}, E_{7}, E_{8}$ and their dual lattices, Proc. Japan Acad., 71 A (1995), pp. 140-143.

[11] - Dixmier's system of projective invariants for a plane quartic, in preparation.

[12] G. Salmon, A Treatise on the Higher Plane Curves, Dublin, 1879 (reprinted by Chelsea Pub. Co, N.Y.).

[13] I. ScHUR, Vorlesungen über Invariantentheorie, Springer-Verlag, 1968. 
